\title{
A CAR-BORNE SAR SYSTEM FOR INTERFEROMETRIC MEASUREMENTS: DEVELOPMENT STATUS AND SYSTEM ENHANCEMENTS
}

\author{
Othmar Frey \\ Gamma Remote Sensing / \\ Earth Observation \& Remote Sensing, \\ ETH Zurich, Switzerland \\ Email: frey@gamma-rs.ch / otfrey@ethz.ch
}

\author{
Charles L. Werner \\ Gamma Remote Sensing \\ Switzerland \\ Email: cw@gamma-rs.ch
}

\author{
Irena Hajnsek, Roberto Coscione \\ Earth Observation \& Remote Sensing, \\ ETH Zurich Switzerland \\ Email: \{hajnsek, coscione\}@ifu.baug.ethz.ch
}

\begin{abstract}
Terrestrial radar systems are used operationally for area-wide measurement and monitoring of surface displacements on steep slopes, as prevalent in mountainous areas or also in open pit mines. One limitation of these terrestrial systems is the decreasing crossrange resolution with increasing distance of observation due to the limited antenna size of the real aperture radar or the limited synthetic aperture of the quasi-stationary SAR systems. Recently, we have conducted a first experiment using a car-borne SAR system at Ku-band, demonstrating the time-domain back-projection (TDBP) focusing capability for the FMCW case and single-pass interferometric capability of our experimental Ku-band car-borne SAR system. The cross-range spatial resolution provided by such a car-based SAR system is potentially independent from the distance of observation, given that an adequate sensor trajectory can be built. In this paper, we give (1) an overview of the updated system hardware (radar setup and high-precision combined INS/GNSS positioning and attitude determination), and (2) present SAR imagery obtained with the updated prototype $\mathrm{Ku}$-band car-borne SAR system.
\end{abstract}

Index Terms-Synthetic aperture radar (SAR), groundbased SAR system, SAR imaging, SAR interferometry, car-borne SAR, CARSAR, GPU, CUDA, parallelization, azimuth focusing, interferometry, Ku-band

\section{INTRODUCTION}

Today, terrestrial radar systems are used operationally for area-wide measurement and monitoring of surface displacements [1], [2] on steep slopes, as prevalent in mountainous areas or also in open pit mines. Terrestrial radar systems are complementary to high-precision point-wise measurements, as obtained with total station theodolites, and also to photogrammetry or laser scanning that typically provide larger coverage but are less sensitive to detect small surface displacements. In addition, terrestrial radar systems are also complementary to spaceborne SAR interferometry based displacement measurements in terms of the viewing geometry and the temporal sampling of the deformation signal.
One limitation of these terrestrial systems is the decreasing cross-range resolution with increasing distance of observation due to the limited antenna size of the real aperture radar or the limited synthetic aperture of the quasi-stationary SAR systems. Recently, we have conducted a first experiment using a car-borne SAR system at $\mathrm{Ku}$-band, presented in [3], demonstrating the time-domain back-projection (TDBP) focusing capability for the frequency-modulated continuous-wave (FMCW)-case and single-pass interferometric capability of our experimental $\mathrm{Ku}$-band car-borne SAR system (see Table I). Most importantly - and in contrast to the deteriorating cross-range resolution of the stationary groundbased systems - the spatial resolution provided by such a car-based SAR system is potentially independent from the distance of observation, given that an adequate sensor trajectory can be built.

Meanwhile, the car-borne measurement setup has been enhanced with a high-precision positioning and attitude determination system, a combined inertial navigation system (INS) and Global Positioning Satellite System (GNSS) system (see Fig. 1). The specifications of the INS/GNSS system are given in Table II. While stationary systems are bound to relatively high frequencies (many of them operate at $\mathrm{Ku}$-band around $17.2 \mathrm{GHz}$ ) to ensure an acceptable cross-range resolution, the proposed carborne system will eventually operate at a lower frequency (L-band) while still achieving a much better cross-range resolution (order of a few decimeters), than stationary terrestrial radar/SAR systems.

In this paper, we give (1) an overview of the updated system hardware (radar, and high-precision combined INS/GNSS positioning and attitude determination), and (2) present SAR imagery obtained with the updated $\mathrm{Ku}$ band car-borne SAR system.

\section{MEASUREMENT SETUP}

Table I contains the specifications of the car-borne FMCW radar prototype system at $\mathrm{Ku}$-band. The current 


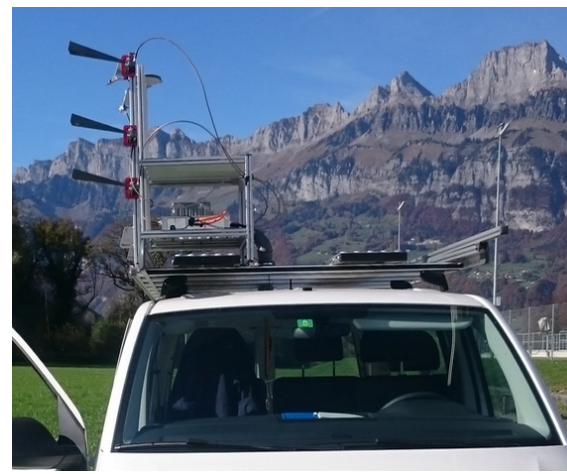

(a)

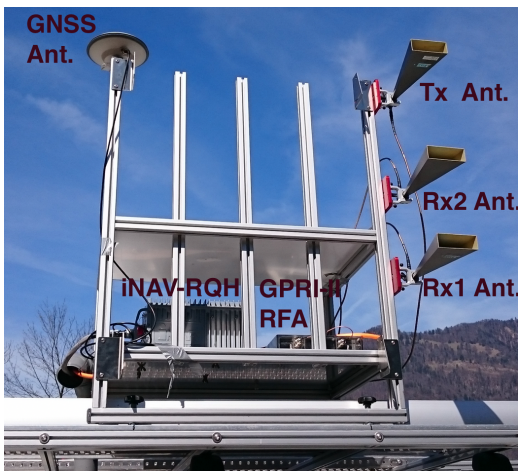

(b)

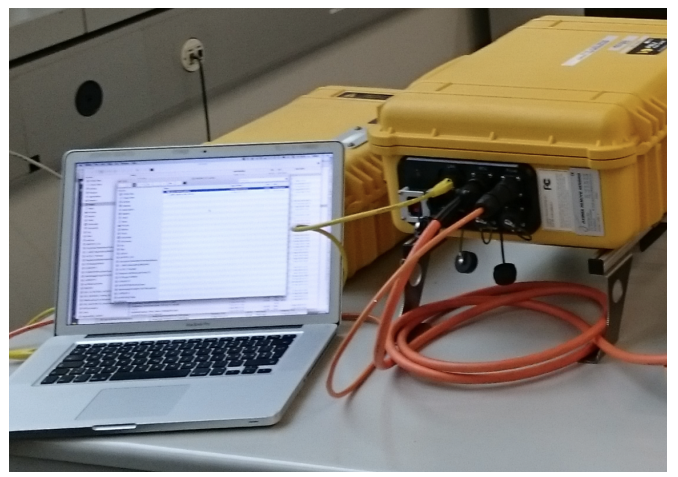

(c)

Fig. 1. a) Front view of the antenna rack with mounted Ku-band horn antennas. b) Sideview of rack hosting one transmit antenna and two receive antennas, the RFA unit of the Ku-band radar, the INS/GNSS iNAV-RQH system with GNSS antenna for high-precision positioning/attitude determination. c) External computer and Instrument Controller Case containing the instrument computer, power supplies, and Software Defined Radio (SDR).

TABLE I

KU-BAND CAR-BORNE SAR SPECIFICATIONS

\begin{tabular}{lc}
\hline \hline Carrier frequency & $17.2 \mathrm{GHz}$ \\
Chirp bandwidth & $50-200 \mathrm{MHz}$ \\
Type & FMCW \\
Chirp lengths & $250 \mu \mathrm{s}-8 \mathrm{~ms}$. \\
ADC sampling rate & $6.25 \mathrm{MHz}$ \\
Elev. beamwidth (3dB) & $25.0 \mathrm{deg}$ \\
Azim. beamwidth (3dB) & $12.5 \mathrm{deg}$ \\
Elev. pointing angle & $15.0 \mathrm{deg}$ (variable) \\
\hline \hline
\end{tabular}

TABLE II

SPECIFICATIONS OF THE IMAR INAV-RQH INS/GNSS SYSTEM

\begin{tabular}{ll}
\hline \hline Positioning accuracy & $2 \mathrm{~cm}$, postproc, RTK/INS \\
& $0.6 \mathrm{~nm} / \mathrm{hr}$, free inertial
\end{tabular}

Attitude accuracy $\quad 0.002 \mathrm{deg}$, postproc. RTK/INS

True heading

Velocity accuracy

0.005 deg postproc. RTK/INS

Gyro. type

$<2 \mathrm{~mm} / \mathrm{s}$ postproc. RTK/INS

Acc. type

3 ring-laser gyros (RLG)

3 servo accelerometers

prototype is a modified configuration of the Gamma Portable Radar Interferometer (GPRI-II) [4]. In Table II the specifications of the high-precision iMAR iNAV$\mathrm{RQH}$ ring-laser gyro INS/GNSS navigation system are given. In Fig. 1 the updated car-borne measurement setup is depicted with an aluminium rack hosting the radar transmit and receive horn antennas, the RFA unit of the Ku-band radar, and the INS/GNSS iNAV-RQH system with the GNSS antenna for high-precision positioning/attitude determination.

\section{GPU-BASED TDBP PROCESSING}

The SAR data is focused using a time-domain backprojection approach [5], [6] adapted to FMCW systems [7]-[9]. Also a graphics processing unit (GPU)-based parallelized implementation of a TDBP algorithm [5], [6] based on NVIDIA's Compute Unified Device Architecture (CUDA) application programming interface had been implemented; a brief performance analysis of a first implementation was given in [8]. A refined efficient parallelized interpolation scheme and block processing is employed to achieve image-focusing times down to the order of 10-15 min for a raw data set of dimensions 3000-by-40000.

\section{RESUlts}

In Fig. 2 a geocoded multi-look intensity (MLI) image of a car-borne SAR data acquisition taken on a road along a section of $285 \mathrm{~m}$ of length is shown. The radar sensor used is a modified configuration (as shown in Fig. 1) of the GPRI-II Ku-band FMCW radar [4] with motion data acquired by means of the highprecision iNAV-RQH INS/GNSS system, the specification of which is given in Table II. Although a highprecision INS/GNSS has been used for the experiment, the short wavelength of the prototype Ku-band system in combination with the rather long synthetic aperture integration time requires that autofocus techniques are 

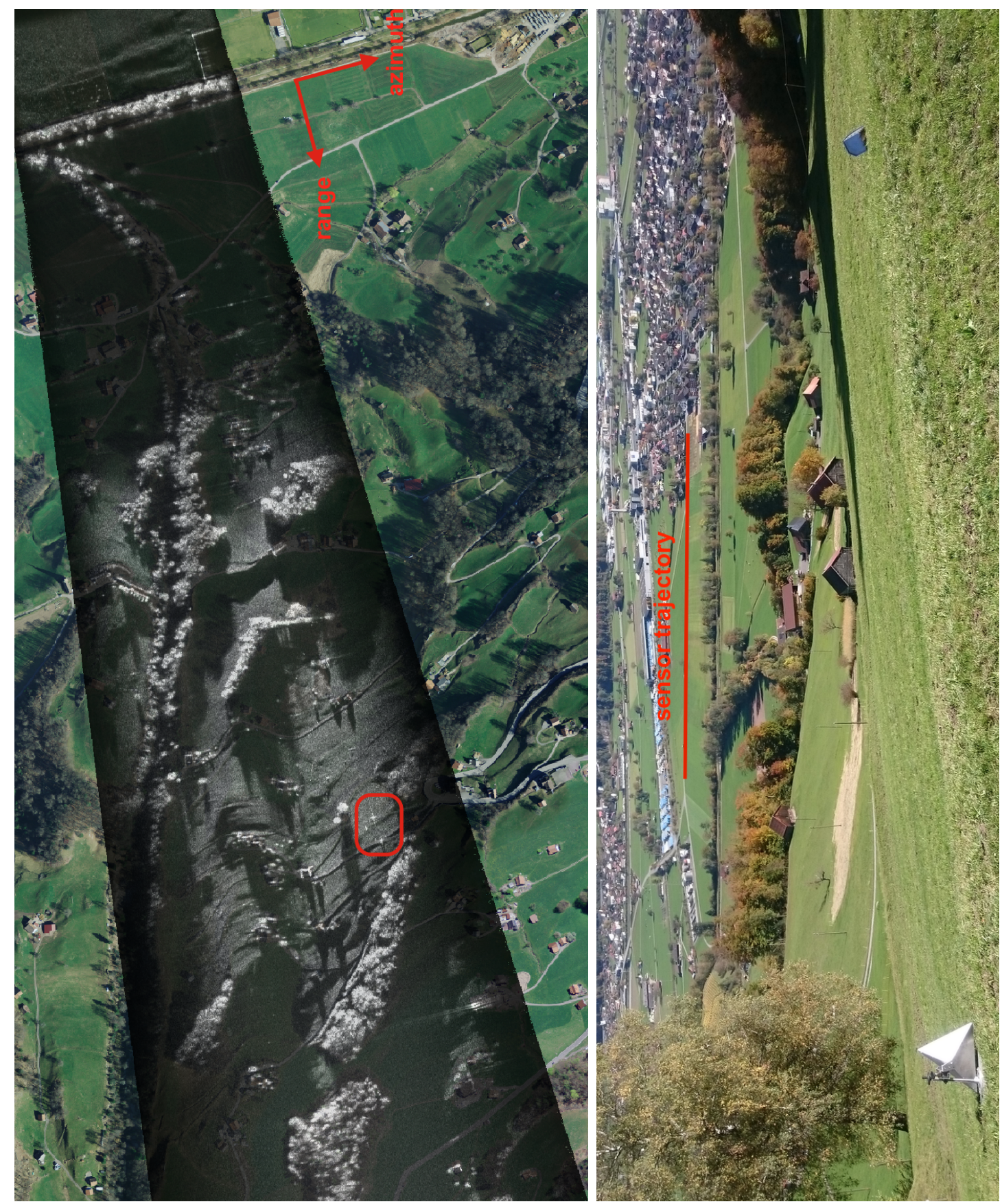

Fig. 2. Left: Geocoded multi-look intensity (MLI) image of a car-borne SAR data acquisition taken on a road along a section of $285 \mathrm{~m}$ of length. The range and azimuth direction as well as the region, where the corner reflectors were deployed, are indicated. Sensor: Modified GPRI-II Ku-band FMCW radar in car-borne configuration (Fig. 1). Processing: Time-domain Back-projection on a NVIDIA Tesla K20 GPU. The data set was focused directly to map coordinates using a DEM. The MLI image is blended with a high-resolution (25cm) orthophoto (Orthophoto: swissimage $25 \mathrm{~cm}$ (C) swisstopo). Right: View from the reflector location on the hillside down to the valley floor where the car was driven along a road to acquire the SAR data set. The sensor trajectory of the car-borne SAR is indicated with a red line.

applied to mitigate unknown residual motion errors. In Fig. 3 an impulse response plot is shown as obtained from one of the corner reflectors deployed in the field. The $3 \mathrm{~dB}$ resolutions in range and azimuth are about $1.8 \mathrm{~m}$ (at a reduced bandwidth of $100 \mathrm{MHz}$ plus a tapering window) and about $10 \mathrm{~cm}$, respectively. The high resolution in azimuth is due to the relatively small extension of the Ku-band horn antennas used in this experiment. Thus, substantial multilooking can be applied in azimuth while keeping the range resolution at an acceptable level. 


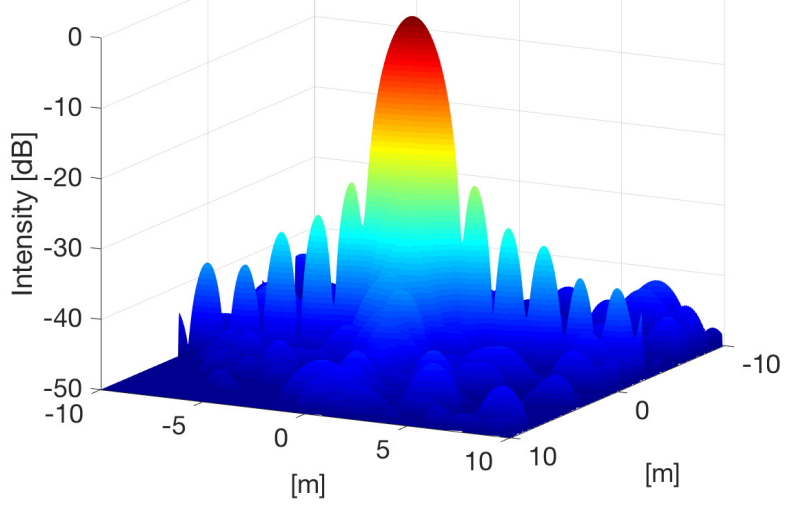

(a)

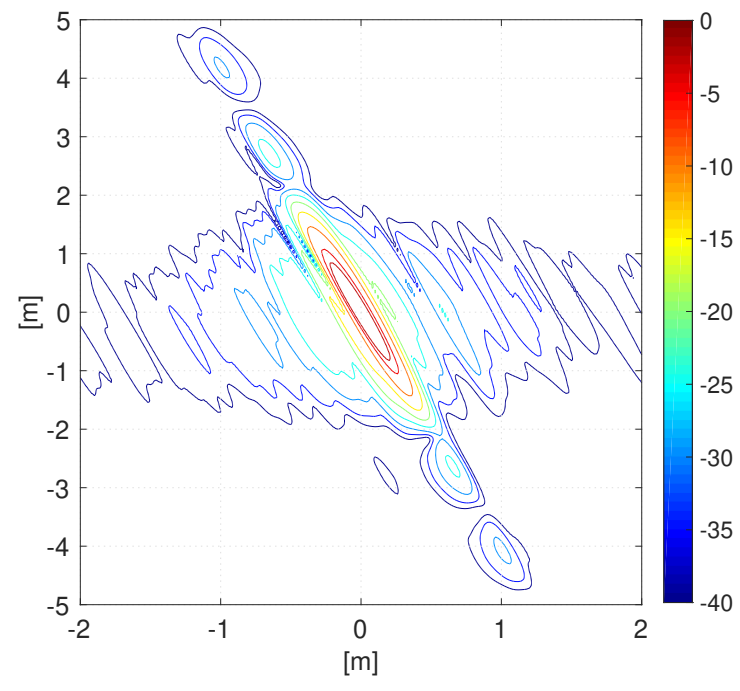

(b)

Fig. 3. Impulse response function (IRF) of a trihedral corner reflector deployed in the field. Since, in this case, the image data grid is directly in map coordinates the IRF is slightly rotated. Note the rather different $3 \mathrm{~dB}$ resolutions of roughly $1.8 \mathrm{~m}$ in range (obtained with $100 \mathrm{MHz}$ bandwidth and a tapering window) and about $10 \mathrm{~cm}$ in azimuth, respectively.

\section{CONCLuSion}

An overview of the current development status of an experimental car-borne SAR system was given including high-resolution focused SAR data obtained with the current prototype Ku-band radar setup. Performance indicators for the resolution and focusing characteristics of the radar and processing system, using a GPUbased TDBP approach, were shown based on an impulse response as obtained from a corner reflector placed in the field. The focus of further developments is on testing the repeat-pass InSAR capability of the car-borne SAR system using a newly developed L-band FMCW radar. In addition, further investigations regarding potential improvements in the measurement and positioning setup are envisaged in view of the targeted repeat-pass interferometric applications.

\section{ACKNOWLEDGMENT}

This joint academic-industry research project is partially funded by the Swiss Commission of Technology and Innovation CTI / Innosuisse.

\section{REFERENCES}

[1] O. Monserrat, M. Crosetto, and G. Luzi, "A review of groundbased SAR interferometry for deformation measurement," ISPRS Journal of Photogrammetry and Remote Sensing, vol. 93, pp. 40-48, 2014.
[2] R. Caduff, F. Schlunegger, A. Kos, and A. Wiesmann, "A review of terrestrial radar interferometry for measuring surface change in the geosciences," Earth Surface Processes and Landforms, vol. 40, no. 2, pp. 208-228, 2015.

[3] O. Frey, C. L. Werner, U. Wegmuller, A. Wiesmann, D. Henke, and C. Magnard, "A car-borne SAR and InSAR experiment," in Proc. IEEE Int. Geosci. Remote Sens. Symp., 2013, pp. 93-96.

[4] C. L. Werner, A. Wiesmann, T. Strozzi, A. Kos, R. Caduff, and U. Wegmuller, "The GPRI multi-mode differential interferometric radar for ground-based observations," in Proc. EUSAR 2012 - 9th European Conference on Synthetic Aperture Radar, Apr. 2012, pp. 304-307.

[5] O. Frey, C. Magnard, M. Rüegg, and E. Meier, "Focusing of airborne synthetic aperture radar data from highly nonlinear flight tracks," IEEE Trans. Geosci. Remote Sens., vol. 47, no. 6, pp. 1844-1858, June 2009.

[6] O. Frey, E. Meier, and D. Nüesch, "Processing SAR data of rugged terrain by time-domain back-projection," in SPIE Vol. 5980: SAR Image Analysis, Modeling, and Techniques X, 2005.

[7] A. Ribalta, "Time-domain reconstruction algorithms for FMCWSAR," IEEE Geoscience and Remote Sensing Letters, vol. 8, no. 3, pp. 396-400, May 2011.

[8] O. Frey, C. L. Werner, and U. Wegmuller, "GPU-based parallelized time-domain back-projection processing for agile SAR platforms," in Proc. IEEE Int. Geosci. Remote Sens. Symp., July 2014, pp. 1132-1135.

[9] C. Stringham and D. G. Long, "GPU processing for UAS-based LFM-CW stripmap SAR," Photogrammetric Engineering \& Remote Sensing, vol. 80, no. 12, pp. 1107-1115, 2014. 